\title{
Uso de medicamentos e o acompanhamento farmacêutico no tratamento de pacientes com fibromialgia
}

\author{
Use of medications and pharmaceutical monitoring in the treatment of patients with fibromyalgia \\ Uso de medicamentos y monitorización farmacéutica en el tratamiento de pacientes con
}

fibromialgia

Recebido: 15/11/2021 | Revisado: 25/11/2021 | Aceito: 30/11/2021 | Publicado: 04/12/2021
Emanuel Cadena da Silva
ORCID: https://orcid.org/0000-0002-1411-3503
Universidade Nilton Lins, Brasil
E-mail: emanuelcadena98@gmail.com
Erick Frota Gomes Figueiredo
ORCID: https://orcid.org/0000-0002-6127-0544
Universidade Nilton Lins, Brasil
E-mail: erick.figueiredo@uniniltonlins.edu.br
Omero Martins Rodrigues Júnior
ORCID: https://orcid.org/0000-0002-8552-3278
Universidade Nilton Lins, Brasil
E-mail: omero.junior@uniniltonlins.edu.br

\begin{abstract}
Resumo
Esta pesquisa tem como objetivo compreender como o acompanhamento farmacêutico pode ajudar no tratamento de fibromialgia, levando em consideração os principais tipos de tratamentos medicamentosos e o papel do farmacêutico. Trata-se de uma revisão integrativa, onde a coleta de dados foi realizada com artigos entre 2016 à 2021 nas bases de dados: Literatura Latino-Americano e do Caribe em Ciências da Saúde (LILACS), Publisher Medline (PUBMED), Scientific Electronic Library Online (SciELO), utilizando-se os seguintes descritores e palavras-chave: Fibromialgia, Dores, Medicamentos e Tratamento. Por meio dos resultados obtidos verificou-se que a melhor opção para o tratamento da fibromialgia é a combinação intervenções farmacológicas e não farmacológicas, com ênfase em tratamentos combinados com medicamentos direcionados a múltiplos mecanismos moleculares, como prediz o Conselho Federal de Farmácia. Por intermédio dos dados discutidos pode-se concluir que a combinação entre terapia psicológica, física e o uso de fármacos apresenta-se como sendo a melhor opção para o tratamento de pacientes com fibromialgia. Nesse sentido, a farmacologia contribui com os medicamentos com finalidade de atingir as vias centrais da dor, favorecendo o paciente e reduzindo sua aflição diária. Contudo, ainda assim se faz necessário uma pesquisa mais aprofundada sobre as alternativas de tratamento para essa condição complexa.
\end{abstract}

Palavras-chave: Fibromialgia; Dor; Combinação de medicamentos; Tratamento farmacológico.

\begin{abstract}
This research aims to understand how pharmaceutical follow-up can help in the treatment of fibromyalgia, taking into account the main types of drug treatments and the role of the pharmacist. This is an integrative review, where data collection was performed with articles between 2016 to 2021 in the following databases: Latin American and Caribbean Literature on Health Sciences (LILACS), Publisher Medline (PUBMED), Scientific Electronic Library Online (SciELO), using the following descriptors and keywords: Fibromyalgia, Pain, Medicines and Treatment. Through the results obtained, it was found that the best option for the treatment of fibromyalgia is the combination of pharmacological and non-pharmacological interventions, with an emphasis on combined treatments with drugs aimed at multiple molecular mechanisms, as predicted by the Federal Council of Pharmacy. Through the data discussed, it can be concluded that the combination of psychological and physical therapy and the use of drugs is the best option for the treatment of patients with fibromyalgia. In this sense, pharmacology contributes with medications with the purpose of reaching the central pain pathways, favoring the patient and reducing their daily affliction. However, even so, further research is needed on treatment alternatives for this complex condition.
\end{abstract}

Keywords: Fibromyalgia; Pain; Drug combinations; Drug therapy.

\section{Resumen}

Esta investigación tiene como objetivo comprender cómo el seguimiento farmacéutico puede ayudar en el tratamiento de la fibromialgia, teniendo en cuenta los principales tipos de tratamientos farmacológicos y el papel del farmacéutico. Se trata de una revisión integradora, donde se realizó la recolección de datos con artículos entre 2016 a 2021 en las 
siguientes bases de datos: Literatura Latinoamericana y del Caribe en Ciencias de la Salud (LILACS), Publisher Medline (PUBMED), Scientific Electronic Library Online (SciELO), utilizando el siguientes descriptores y palabras clave: Fibromialgia, Dolor, Medicamentos y Tratamiento. A través de los resultados obtenidos, se encontró que la mejor opción para el tratamiento de la fibromialgia es la combinación de intervenciones farmacológicas y no farmacológicas, con énfasis en tratamientos combinados con fármacos dirigidos a múltiples mecanismos moleculares, tal y como predice el Consejo Federal de Farmacia. A través de los datos comentados se puede concluir que la combinación de fisioterapia y psicoterapia y el uso de fármacos es la mejor opción para el tratamiento de pacientes con fibromialgia. En este sentido, la farmacología contribuye con medicamentos con el propósito de llegar a las vías centrales del dolor, favoreciendo al paciente y reduciendo su aflicción diaria. Sin embargo, aun así, se necesitan más investigaciones sobre las alternativas de tratamiento para esta compleja afección.

Palabras clave: Fibromialgia; Dolor; Combinación de medicamentos; Tratamiento farmacológico.

\section{Introdução}

A fibromialgia (FM) é uma condição clínica caracterizada por sensibilidade atípica do sistema nervoso central a estímulos externos. Geralmente afeta entre 2 e $8 \%$ da população total, com forte predominância do sexo feminino (Tan, Aaniste, Champion, 2019).

A característica clínica mais reconhecível associada à fibromialgia é a dor generalizada e a sensibilidade em várias regiões do corpo, na ausência de patologia nos locais de dor. Os pacientes também podem apresentar uma grande variedade de outros sintomas, incluindo fadiga e distúrbios do sono, alterações cognitivas, como baixa concentração e memória, e sistemas sensoriais amplificados que levam a uma intolerância a ruídos altos, luzes brilhantes e odores fortes (Marques, et al., 2018).

Neste sentido, é importante ressaltar que a fibromialgia está associada a várias condições médicas interrelacionadas, incluindo a síndrome do intestino irritável (SII), disfunção da articulação temporomandibular, dores de cabeça tensionais, síndrome da fadiga crônica e síndrome das pernas inquietas (Ribeiro, 2016; Ammad, 2018).

Na concepção de Júnior \& Almeida (2018), a fisiopatologia da fibromialgia é complexa, embora a compreensão tenha aumentado exponencialmente nos últimos anos. O efeito líquido de vários fatores leva a uma sensibilização da dor central e dos centros de processamento sensorial, de modo em que os pacientes se tornem excessivamente sensíveis a estímulos externos. Nesse sentido, estudos de imagem funcional têm mostrado respostas amplificadas em regiões sensoriais do cérebro quando estímulos mecânicos ou dolorosos são administrados.

Nesse contexto, Vilaça et al., (2020) elucida conexões aprimoradas entre os centros cerebrais que processam a dor e estímulos sensoriais, como o córtex insular, e partes do cérebro associadas à concentração e à memória de trabalho, como a rede de atenção executiva frontoparietal. Isso acaba por fornecer alguma explicação para os sintomas cognitivos que muitos pacientes apresentam. Várias anormalidades de neurotransmissores também foram identificadas em pacientes com fibromialgia (KIA \& CHOY, 2017) e estão relacionadas à modulação das vias inibitórias sensoriais descendentes do cérebro à medula espinhal. Estes são discutidos em relação a intervenções farmacológicas específicas em seções posteriores.

É nesse cenário que surgem os fármacos, apresentando-se como um agente de grande importância para colaborar no tratamento da FM, a fim de minimizar os sintomas, para que os pacientes possam participar de modalidades não medicamentosas que forneçam tratamento de longo prazo para a doença, como exercícios e atividades comportamentais, lembrando que as terapias não medicamentosas devem ser usadas sempre que possível (Portuguez \& Gallardo, 2017; Sagy, et al., 2019).

Entre os medicamentos com efeito analgésico que são úteis no controle da FM destacam-se o paracetamol (Tylenol@), que de acordo com a literatura é utilizado como AINEs (antiinflamatórios não esteroidais), assim como o ibuprofeno (Advilß, Motrilß) e a aspirina em combinação com outros medicamentos que servem para aliviar os desconfortos causados pela fibromialgia, além dos antidepressivos, anticonvulsivantes, agonistas da dopamina e hormônio do crescimento. Sem contar com os inibidores seletivos da recaptação da serotonina (SSRIs) ou a combinação de ambos que produzem uma melhora leve a moderada nos sintomas da fibromialgia (Ziani, et al., 2017). 
Deste modo, esta pesquisa é de inteira relevância para a área acadêmica, pois, retrata justamente a importância de um profissional habilitado, a saber, o farmacêutico, para atender o paciente quando ele entra numa farmácia, nesse sentido, é preciso compreender o paciente patologicamente, sendo necessário que ele faça uma anamnese para verificar se o tratamento que o paciente está fazendo está auxiliando na melhora de seu quadro de saúde (Oliveira Jr \& Almeida, 2018; CFF, 2020).

Assim, se faz necessário que o farmacêutico tenha um olhar atento principalmente quanto a utilização de medicamentos constantes para a dor, particularmente se ele detecta que o cliente/paciente é diagnosticado com fibromialgia, já que a interação entre vários medicamentos para a dor pode trazer sérias consequências para o cliente/paciente, desta forma o acompanhamento farmacêutico começa numa simples conversa com o paciente, sendo que através dela é possível orientar da melhor forma possível (Vilaça, et al., 2020).

Dessa maneira, a presente pesquisa tem como objetivo compreender como o acompanhamento farmacêutico pode ajudar no tratamento de fibromialgia, levando em consideração os principais tipos de tratamentos medicamentosos e o papel do profissional farmacêutico frente aos indivíduos acometidos pela doença.

\section{Metodologia}

\subsection{Tipo de Estudo}

Este trabalho consiste numa pesquisa qualitativa, na modalidade revisão integrativa de literatura. Em que o método possibilita busca, análise crítica e síntese do conhecimento produzido sobre o tema investigado podendo agregar estudos de diversas metodologias. Desse modo, permite a identificação de lacunas de conhecimento, direcionamento para futuras pesquisas e a incorporação da aplicabilidade de resultados significativos na prática (Marconi \& Lakatos, 2017).

\subsection{Coleta de Dados}

A coleta de dados foi realizada com artigos entre 2016 à 2021 nas bases de dados: Literatura Latino-Americano e do Caribe em Ciências da Saúde (LILACS), Publisher Medline (PUBMED), Scientific Electronic Library Online (SciELO), utilizando-se os seguintes descritores e palavras-chave: Fibromialgia, Dores, Medicamentos e Tratamento. Onde foram selecionados 80 artigos de maior destaque, dos quais 14 corresponderam aos critérios de inclusão deste estudo.

\subsection{Análise de Dados}

Os critérios de inclusão adotados foram: artigos de pesquisa original publicados de forma completa, livre e gratuita em periódicos disponíveis nas bases de dados selecionadas, nos idiomas português, inglês e espanhol, condizentes com o objetivo proposto e os descritores e/ou palavras-chave listados no protocolo previamente validado. Já os critérios de exclusão adotados foram: Os artigos que estavam em mais de uma base de dados foram considerados duplicatas e automaticamente excluídos, ao mesmo tempo fora do período de análise. Por fim, obteve-se 10 artigos relevantes para o tema proposto.

\section{Resultados e Discussão}

Por meio dos dados apresentados no quadro integrativo (Quadro 1), pôde-se inferir que os medicamentos mais usados no tratamento da fibromialgia, que oportunizam uma elevada satisfação quanto aos resultados obtidos são a pregabalina e a duloxetina (Poluhal \& Grosmann, 2018), onde a pregabalina atua como um modulador do canal de cálcio, que minimiza a liberação de neurotransmissores excitatórios da dor nas terminações nervosas (substância P) e o glutamato, proporcionado um alivio significativo da dor, fadiga, ansiedade e também de distúrbios do sono. Já a duloxetina, atua como inibidor da receptação da serotonina e da noradrenalina que também vem sendo eficaz na diminuição da dor, favorecendo a melhora da capacidade 
funcional na fibromialgia, independentemente da presença de depressão.

Quadro 1. Artigos usados para a elaboração da revisão integrativa.

\begin{tabular}{|c|c|c|c|}
\hline Base de dados & Título do artigo & Autor/Ano & Considerações / Temática \\
\hline SciELO & $\begin{array}{l}\text { Avaliação da ingestão alimentar } \\
\text { e qualidade de vida de mulheres } \\
\text { com fibromialgia }\end{array}$ & Batista et al., 2016 & $\begin{array}{l}\text { A combinação dos antioxidantes encontrados nas } \\
\text { vitaminas e minerais com analgésicos podem reduzir } \\
\text { as doses desses medicamentos e como consequência } \\
\text { melhorar a sensação de dor das pacientes com FM. }\end{array}$ \\
\hline SciELO & $\begin{array}{l}\text { Novas diretrizes para o } \\
\text { diagnóstico da fibromialgia }\end{array}$ & Heymann et al., 2017 & $\begin{array}{l}\text { O Índice de Dor Generalizada é significativamente } \\
\text { superior nos pacientes com FM, quando comparado } \\
\text { com outras situações clínicas como lúpus eritematoso } \\
\text { sistêmico, osteoartrite e artrite reumatoide. }\end{array}$ \\
\hline BVS & $\begin{array}{l}\text { Atualização nas Diretrizes de } \\
\text { Tratamento na Síndrome de } \\
\text { Fibromialgia com Foco na } \\
\text { Farmacologia }\end{array}$ & Kia \& Choy, 2017 & $\begin{array}{l}\text { Sem evidências para apoiar o uso de múltiplas terapias } \\
\text { para SFM. } \\
\text { Licenciamento devem ser tratados pelos órgãos } \\
\text { reguladores, especialmente quando houver cuidados } \\
\text { com o uso de terapias fora da licença. }\end{array}$ \\
\hline LILACS & $\begin{array}{l}\text { A pregabalina melhora os } \\
\text { distúrbios do sono na } \\
\text { fibromialgia? }\end{array}$ & Poluhal et al.,2018 & $\begin{array}{l}\text { A PG é um fármaco eficiente e seguro no manuseio } \\
\text { dos distúrbios do sono em pacientes com FM devido à } \\
\text { diminuição do número de despertares e aumento do } \\
\text { tempo de duração do sono. }\end{array}$ \\
\hline LILACS & $\begin{array}{l}\text { Fibromialgia: O corpo, a mente } \\
\text { e o estigma }\end{array}$ & Ribeiro, 2016 & $\begin{array}{l}\text { Abordagem multidisciplinar com recurso a diferentes } \\
\text { tipos de terapia e com a participação ativa do doente. } \\
\text { O tratamento farmacológico melhora a qualidade de } \\
\text { vida e deve ser escolhido de acordo com os sintomas } \\
\text { presentes. }\end{array}$ \\
\hline LILACS & $\begin{array}{l}\text { A prevalência de fibromialgia: } \\
\text { atualização da revisão de } \\
\text { literatura }\end{array}$ & Santo et al., 2017 & $\begin{array}{l}\text { Esta atualização de revisão de literatura mostra um } \\
\text { aumento expressivo de estudos de prevalência da FM } \\
\text { ao redor do mundo. }\end{array}$ \\
\hline SciELO & $\begin{array}{l}\text { A prevalência da fibromialgia } \\
\text { no Brasil - estudo de base } \\
\text { populacional com dados } \\
\text { secundários da pesquisa de } \\
\text { prevalência de dor crônica } \\
\text { brasileira } \\
\end{array}$ & Souza et al., 2018 & $\begin{array}{c}\text { A prevalência da síndrome de fibromialgia foi } \\
\text { estimada em } 2 \% \text { da população brasileira, através de } \\
\text { dados secundários a um estudo de prevalência da dor } \\
\text { crônica. }\end{array}$ \\
\hline BVS & $\begin{array}{c}\text { Dor crônica generalizada e } \\
\text { síndrome de fibromialgia: } \\
\text { marcadores de risco ao longo da } \\
\text { vida em jovens }\end{array}$ & Tan et al., 2019 & $\begin{array}{l}\text { Marcadores de risco ao longo do curso de vida pode } \\
\text { fornecer uma visão sobre porque um jovem pode } \\
\text { adquirir CWP cumprindo os critérios para SFM. Seus } \\
\text { marcadores de risco eram história familiar, nascimento } \\
\text { prematuro, adversidade na infância, hipermobilidade } \\
\text { das articulações, obesidade leve, ansiedade e } \\
\text { depressão, múltiplos distúrbios de dor primária ou } \\
\text { funcional, trauma físico, deficiência de ferro e doença } \\
\text { infecciosa com fadiga crônica. }\end{array}$ \\
\hline BVS & $\begin{array}{l}\text { Novos medicamentos para } \\
\text { tratamento de fibromialgia }\end{array}$ & Vilaça et al., 2020 & $\begin{array}{l}\text { Necessidade de um acompanhamento individualizado } \\
\text { e exclusivamente sintomático. Uso de medicamentos já } \\
\text { usados e de novos fármacos para tratamento dos } \\
\text { sintomas da doença associado a intervenção não } \\
\text { farmacológica. }\end{array}$ \\
\hline SciELO & $\begin{array}{l}\text { Revisões de } 2016 \text { para os } \\
\text { critérios diagnósticos de } \\
\text { fibromialgia de } 2010 / 2011\end{array}$ & Wolfe et al., 2016 & $\begin{array}{l}\text { Os critérios de fibromialgia têm boa sensibilidade e } \\
\text { especificidade. Esta revisão combina os critérios do } \\
\text { médico e do questionário, minimiza a classificação } \\
\text { incorreta de distúrbios regionais de dor e elimina a } \\
\text { recomendação anteriormente confusa sobre exclusões } \\
\text { diagnósticas. Os critérios baseados no médico são } \\
\text { válidos para o diagnóstico individual do paciente. }\end{array}$ \\
\hline
\end{tabular}

Fonte: Dados da pesquisa integrativa (2021). 
De acordo com as literaturas apresentadas no quadro integrativo verifica-se que as terapias farmacológicas existentes na atualidade para o tratamento da fibromialgia incluem quatro classes extensas de medicamentos, sendo eles: drogas antiepiléticas, antidepressivos tricíclicos, inibidores seletivos da recaptação de serotonina (ISRS) e inibidores da recaptação de serotonina noradrenalina (CFF, 2020; Vilaça, et al., 2020).

Em uma visão mais detalhada, segundo denotam Júnior e Almeida (2018), Souza e Perissinotti (2018) podem ser usados outros tipos de fármacos para tratar os sintomas da fibromialgia, dentre eles estão os relaxantes musculares, antagonistas dos receptores 5-HT3, agonistas dopaminérgicos, antioxidantes, benzodiazepínicos, antiinflamatórios esteroidais e não esteroidais e analgésicos (CFF, 2020).

Wolfe et al. (2016), Tan Jaaniste e Chapion (2019) e Costa, Bezerra e Paula (2021) relatam que nos Estados Unidos, somente a pregabalina (anticonvulsivante), a duloxetina (antidepressivo) e o milnaciprano (antidepressivo) são aprovados pelo Food and Drug Administration (FDA) para o tratamento da fibromialgia, sendo considerado off label (não seguem as indicações homologadas para o uso do fármaco) a utilização de todos os outros medicamentos.

Para Batista et al. (2016), Marques et al. (2017), Ziani et al. (2017) e Azevedo (2018), a serotonina e a noradrenalina (neurotransmissores excitatórios) também se apresentam com eficiência satisfatória produzindo efeito analgésico, assim como a amitriptilina. Já a mirtazapina (um antagonista alfa-2 de ação pré-sináptica central) auxilia no tratamento da fibromialgia, visando diminuir os sintomas, principalmente da dor, podendo ajudar a aumentar a serotonina e a noradrenalina no sistema nervoso central (SNC). Entretanto, de acordo com o Conselho Federal de Farmácia (CFF, 2020), a melhor alternativa continua sendo o uso da pregabalina e duloxetina em relação à terapia individual de cada um desses medicamentos.

Diversos autores (Ribeiro, 2016; Kia \& Choy, 2017; Junior \& Almeida, 2018; Benlidayi, 2019) afirmam que a combinação e uso dos fármacos melhora os resultados clínicos da fibromialgia em comparação com a utilização individual, destacando a relevância de pesquisas que comparem essas e outras combinações no uso de apenas um fármaco. É importante ressaltar que o acompanhamento do farmacêutico nessa etapa é essencial devido a segurança do uso dos medicamentos adequados, a fim de contribuir de forma plausível para o avanço da saúde do paciente.

Para Vilaça et al. (2020) é aceitável na atualidade o fato de que ainda a melhor opção para o tratamento da fibromialgia é a combinação intervenções farmacológicas e não farmacológicas, com ênfase em tratamentos combinados com medicamentos direcionados a múltiplos mecanismos moleculares, como prediz o Conselho Federal de Farmácia (2020).

Nesse sentido, o ideal para dar sequência ao tratamento farmacológico é a leitura dos sintomas em análise (predominantes), sendo necessário o acompanhamento do farmacêutico, a fim do uso plausível e escolha dos fármacos mais apropriados para a diminuição das dores crônicas, como por exemplo: antidepressivos tricíclicos (amitriptilina), a ciclobenzaprina, a gabapentina, os inibidores da recaptação da serotonina e da adrenalina (duloxetina e milnacipran), comprovados cientificamente como os mais eficazes no tratamento de fibromialgia ( Júnior \& Almeida, 2018, Morales, et al., 2019).

Kia e Choy (2017) denota que de uma forma geral as diretrizes devem reconhecer a necessidade do tratamento farmacológico dos sintomas da fibromialgia associado a educação do paciente e a atividade física. Ou seja, aqueles pacientes que não respondem a intervenções não farmacológicas, devem ser acrescentadas terapias farmacológicas em especial àquelas com distúrbios do sono ou do humor. Atualmente, não há evidências para apoiar o uso de múltiplas terapias para a FM, embora isso seja comumente observado na prática clínica.

À vista disso, torna-se evidente que o acompanhamento do farmacêutico no tratamento de pacientes com fibromialgia é de extrema importância, devido a esta ser uma doença que acomete uma grande parte da população, apresentando um tratamento definido pelo Conselho Federal de Farmácia como sendo bastante complexo, devido a presença de várias classes medicamentosas.

Logo, pode-se inferir que todos os dias novos fármacos vêm sendo estudados para serem usados e que contenham um 
número menor de efeitos adversos em indivíduos com fibromialgia. Foram encontradas nos últimos cinco anos, cinco patentes com fármacos de diferentes grupos farmacológicos, mas que buscam amenizar e/ou neutralizar os sintomas da fibromialgia, demonstrando dessa forma que a cada dia se buscam novos estudos e novas ferramentas para tratar a patologia.

\section{Considerações Finais}

Por intermédio dos dados discutidos pode-se concluir que a combinação entre terapia psicológica, física e o uso de fármacos apresenta-se como sendo a melhor opção para o tratamento de pacientes com fibromialgia. Nesse sentido, a farmacologia contribui com os medicamentos com finalidade de atingir as vias centrais da dor, favorecendo o paciente e reduzindo sua aflição diária. Contudo, ainda assim se faz necessário uma pesquisa mais aprofunda sobre as alternativas de tratamento para essa condição complexa.

Na verdade, a fibromialgia atua como uma patologia crônica e dolorosa que possui a etiopatogenia desconhecida, porém acredita-se que fatores ambientais, genéticos e biológicos estão envolvidos na fisiopatologia da doença. Dessa forma, torna-se evidente a importância de estudos nessa área, onde o tratamento requer uma terapia individual para cada paciente, combinando o tratamento farmacológico e o não farmacológico. Mediante a isso, o tratamento farmacológico na fibromialgia caracteriza-se pela associação de diversas classes medicamentosas e é de grande importância, pois tem a finalidade de diminuir os sintomas e/ou melhorar a qualidade de vida dos pacientes acometidos por essa patologia.

Dessa maneira, ao se construir essa pesquisa observou-se a necessidade da intervenção de medidas públicas a fim de proporcionar um maior conhecimento tanto dos profissionais da saúde como da população em geral sobre a Fibromialgia. No tocante, os pacientes não enfrentariam tantas dificuldades com essa patologia. Dada à característica multifatorial dessa condição, faz-se necessário que se tenha uma interferência multiprofissional no tratamento para prolongar a qualidade de vida e bem-estar do(a) paciente.

Nessa circunstância, essa perspectiva justifica-se pela relevância do tema e pela sua colaboração para futuros estudos sobre esse assunto, objetivando elucidar o trabalho do profissional farmacêutico no acompanhamento terapêutico de pacientes com fibromialgia, promovendo o bem-estar do ser cidadão e compreendendo ainda mais sua atuação em meio a sociedade como um todo.

\section{Referências}

Ammad, N. (2018). Comparative Efficacy of Pharmacological and Nonpharmacological Interventions in Managing Primary and Secondary Outcomes of Fibromyalgia Syndrome. Medical Student Research Journal,6 (1), 11-20.

Azevedo, P. M. (2018). A ciência da dor: Sobre fibromialgia e outras síndromes dolorosas persistentes e sobre a natureza humana. Editora Unesp Digital.

Benlidayi, I. C. (2019). Role of inflammation in the pathogenesis and treatment of fibromyalgia. Rheumatology international, 39(5), 781791. https://doi.org/10.1007/s00296-019-04251-6

Batista, E. D. et al. (2016). Avaliação da ingestão alimentar e qualidade de vida de mulheres com fibromialgia. Revista Brasileira de Reumatologia. 56(2), 105110 .

CFF. (2020). Fibromialgia: sensibilidade, diagnóstico e responsabilidade no tratamento e acompanhamento do paciente.https://www.cff.org.br/noticia.php?id=5759. Conselho Federal de Farmácia.

Costa, A. V. F, Bezerra, L. C, \& Paula, J. A (2021). Use of psychotropic drugs in the treatment of fibromyalgia: a systematic review. Journal of Human Growth and Development, 31(2), 336-345.

Junior, J. \& Almeida, M. (2018). The current treatment of fibromyalgia. Brazilian Journal of Pain, 1(3), $255-262$.

Kia, S. \& Choy, E. (2017). Update on Treatment Guideline in Fibromyalgia Syndrome with Focus on Pharmacology. Biomedicines.,5(2):20.

Marconi, M. \& Lakatos, E. (2017). Fundamentos de metodologia científica. (8a ed.), Atlas.

Marques, A. P. et al. (2017). A prevalência de fibromialgia: atualização da revisão de literatura. Revista Brasileira de Reumatismo, 57(4),. 356-363. 
Research, Society and Development, v. 10, n. 16, e104101623355, 2021

(CC BY 4.0) | ISSN 2525-3409 | DOI: http://dx.doi.org/10.33448/rsd-v10i16.23355

Morales, K. O. et. al. (2019). O uso da técnica manual shiatsu no alívio dos sintomas decorrentes da fibromialgia. Unisanta Health Science, 3(1), 1-17.

Oliveira Jr, J. O \& Almeida, M. B. (2018). The current treatment of fibromyalgia. Br J Pain. 1(3):255-62.

Poluhal, R. L \& Grossmann, E. A. (2018). pregabalina melhora os distúrbios do sono na fibromialgia? Br J Pain. 1(2):163-6.

Portuguez, R. \& Gallardo, P. (2017). Caracterización del dolor fibromiofascial, hipótesis etiológica, aplicación y efecto del tratamiento. Revista de la Sociedad Espanola del Dolor, 24(4), 201-210.

Ribeiro, F. N (2016). Fibromialgia: o corpo, a mente e o estigma.

Souza, J. B. \& Perissiotti, D. M. N. (2018). A prevalência da fibromialgia no Brasil - estudo de base populacional com dados secundários da pesquisa de prevalência de dor crônica brasileira. BrJP 1(4): 345-348.

Sagy, I. V. et al. (2019). Safety and efficacy of medical cannabis in fibromyalgia. Journal of clinical medicine, 8(6), 1-12. https://doi.org/10.3390/jcm8060807

Tan, A. C, Aanieste, T, \& Champion, D. (2019). Chronic Widespread Pain and Fibromyalgia Syndrome: Life-Course Risk Markers in Young People. Pain Res Manag. 6584753 .

Vilaça, M. M. O. et al. (2020). Novos medicamentos para o tratamento da fibromialgia. Research, Society and Development, 9(3), e24932308, 2020.

Wolfe F. et al. (2016). Revisions to the 2010/2011 fibromyalgia diagnostic criteria. Semin Arthritis Rheum. 46(3):319-329. 10.1016/j.semarthrit.2016.08.012.

Ziani, M. M. et al. (2017). Efeitos da terapia manual sobre a dor em mulheres com fibromialgia: uma revisão de literatura. Ciência e Saúde, $10(1)$, $48-55.2017$. 